\title{
Search times with arbitrary detection constraints
}

\author{
Daniel Campos, ${ }^{1}$ Frederic Bartumeus, ${ }^{2}$ and Vicenç Méndez ${ }^{1}$ \\ ${ }^{1}$ Grup de Física Estadística, Departament de Física, Facultat de Ciències, Universitat Autònoma de Barcelona, \\ 08193 Bellaterra (Barcelona), Spain \\ ${ }^{2}$ Centre d'Estudis Avançats de Blanes (CEAB-CSIC), Accés Cala Sant Francesc 14, 17300 Blanes, Girona, Spain
}

(Received 16 April 2013; revised manuscript received 9 July 2013; published 2 August 2013)

\begin{abstract}
Random encounters in space are central to describing diffusion-limited reactions, animal foraging, search processes, and many other situations in nature. These encounters, however, are often constrained by the capacity of the searcher to detect and/or recognize its target. This can be due to limited binding and perception abilities of the searcher or hiding and avoiding mechanisms used by the target. Hence detection failure upon passage over the target location turns the process into an $n$-passage problem, with $n$ being random. Here we provide a general description of this detection problem for arbitrary dimensions and arbitrary detection constraints. The mean detection time (MDT) for a random searcher embedded in a sea of homogeneously distributed targets is obtained as a function of the target density $\rho$, the size domain $L$, and the effective detection distance $a$. While the scaling with $\rho$ and $L$ is found to be universal and equivalent to that found for the corresponding first-passage problem, the scaling of the MDT on $a$ depends on the specific detection mechanism considered.
\end{abstract}

DOI: 10.1103/PhysRevE.88.022101

PACS number(s): 02.50.-r, 87.10.-e, 05.40.Fb

\section{INTRODUCTION}

First-passage time statistics have been used recurrently to describe the random encounter of a certain particle or individual with its target [1,2], for instance, in diffusionlimited reaction kinetics [3], animal foraging [4-7], searching for specific network nodes [8,9], search and rescue operations [10], and ion transport [11]. General expressions have been provided for the mean first-passage time (MFPT) of a random walker moving in regular lattices [12,13] as well as in scale-invariant media of arbitrary dimension [8]. The case of persistent walkers has also been addressed recently [14], thus facilitating the application of these approaches to more sophisticated moving entities rather than classical Brownian walkers. While it is true that under particular considerations the MFPT may be insufficient to provide accurate information of the first-passage statistics [15], this mean value is still used as an appropriate parameter in most practical situations.

The first-passage approach, however, assumes perfect detection upon encounter with the target, while nature is full of examples where such perfect efficiency is far from true. For instance, the reactive or binding surfaces of many molecular structures can only be satisfactorily assembled under the appropriate conditions to overcome energetic barriers, among other restrictions. Often these microscopic details are implicitly considered by introducing effective reaction rates in kinetic models [16], but more rigorously, one should take into account that successful detection (or reaction) can depend explicitly on the direction from which the target is approached, the speed with which it is approached, etc. This is even more evident for random searches in animals or humans; for example, the detection ability of organisms gets reduced as their speed increases [17,18], especially if the targets (e.g., prey) are able to use hiding or other mechanisms to avoid detection. Also, experiments with patrol aircrafts have concluded that the probability to detect visually a target in the open sea decreases with the inverse of the third power of the horizontal distance to it $[19,20]$, a result which has been used for decades for search and rescue planning methods.
These kinds of constrained detection problems have been studied before $[21,22]$ for specific situations (also within the context of imperfect or evanescent trap models [23-25]), but here we show that universal and intuitive conclusions for the mean detection time (MDT) can be reached under very general conditions. We consider (see Fig. 1) a searcher with an effective perception size $a$ (this is a measure of how close to the target the searcher must get in order to make detection possible) moving in a sea of sparse immobile and identical targets homogeneously distributed in space, with a total size domain $L$. So one can define the target density as $\rho \equiv N / L^{d}$, with $N$ being the number of targets and $d$ being the spatial dimension of the system. In the following, we provide a general derivation for the first-detection time probability distribution and its mean value (Sec. II), and then we illustrate in Sec. III the utility of our approach by giving specific expressions for two different detection mechanisms: speed-selective and stop-and-scan mechanisms. Once we have explored how our approach works at practice, we will show that it is possible to provide general scaling laws for the mean detection time as a function of the target density or the size domain for a rather general class of detection mechanisms; this will be done in Sec. IV. Finally, we will finish in Sec. V by summarizing the most relevant conclusions and results from our work.

\section{DERIVATION OF THE MDT}

A general expression for the first-detection time distribution starting from an arbitrary initial position $\mathbf{r}_{0}$ can be derived, provided one knows the probability $q\left(t \mid \mathbf{r}_{0}\right)$ that the searcher will detect its target at time $t$ starting from position $\mathbf{r}_{0}$. To do this, we use an extension of the classical renewal approach for first-passage problems [26,27]; we divide the possible trajectories leading to detection in two classes: (i) those for which this represents the first detection and (ii) those for which this represents the second, third, etc., detection, provided the first detection occurred at a previous time $t^{\prime}$. Therefore, one 


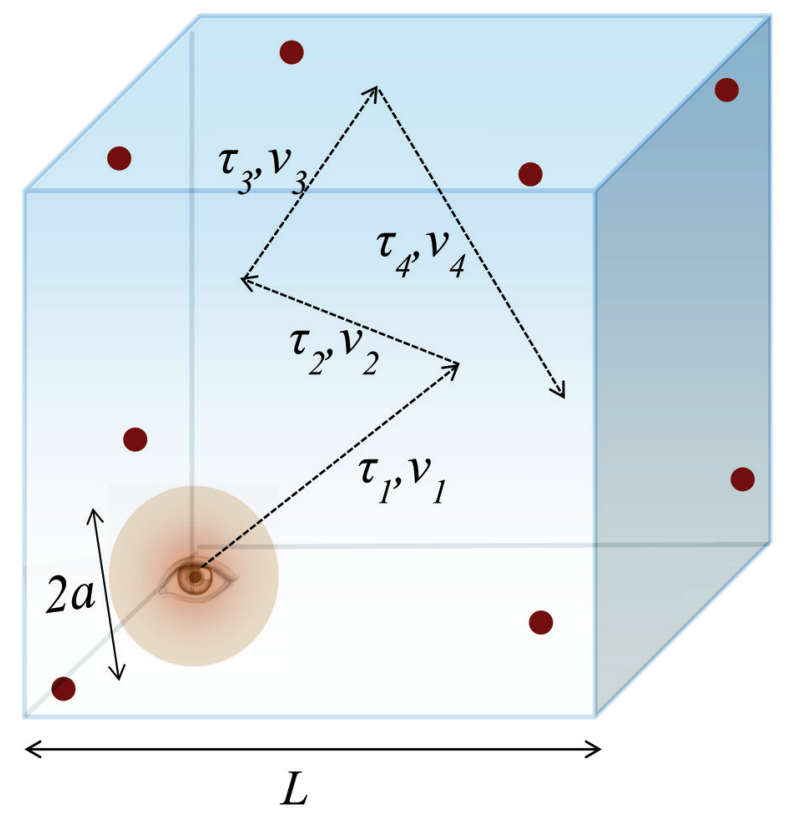

FIG. 1. (Color online) General representation of the nonperfect detection problem, with a random searcher moving in a domain of size $L$ and trying to detect point targets (represented with small dark circles) with an effective detection distance $a$. The searcher is assumed to perform consecutive flights of random duration $\tau_{i}$ and random speed $v_{i}$.

can write for the single-target (i.e., $N=1$ ) case

$$
q\left(t \mid \mathbf{r}_{0}\right)=f\left(t \mid \mathbf{r}_{0}\right)+\int_{0}^{t} q\left(t-t^{\prime} \mid \mathbf{r}_{\text {targ }}\right) f\left(t^{\prime} \mid \mathbf{r}_{0}\right) d t^{\prime},
$$

where $f\left(t \mid \mathbf{r}_{0}\right)$ is the first-detection time distribution and we have assumed in the last term that at time $t^{\prime}$ a new search starts from the target position $\mathbf{r}_{\text {targ }}$, so the memory effects related to the previous trajectory of the searcher are neglected. After Laplace transform in time, the previous expression leads to

$$
f\left(s \mid \mathbf{r}_{0}\right)=\frac{q\left(s \mid \mathbf{r}_{0}\right)}{1+q\left(s \mid \mathbf{r}_{\text {targ }}\right)} .
$$

For the case $N>1$ one needs to generalize (1) to

$$
q\left(t \mid \mathbf{r}_{0}\right)=\sum_{j} f_{j}\left(t \mid \mathbf{r}_{0}\right)+\sum_{j} \int_{0}^{t} q\left(t-t^{\prime} \mid \mathbf{r}_{j}\right) f_{j}\left(t^{\prime} \mid \mathbf{r}_{0}\right) d t^{\prime},
$$

where the index $j$ is used to identify the different targets and $f_{j}\left(t \mid \mathbf{r}_{0}\right)$ is the first-detection time distribution for the $j$ th target. If all targets are identical and regularly spaced, one can divide the volume domain $L^{d}$ into $N$ identical blocks of volume $L^{d} / N=\rho^{-1}$ (and characteristic size $L N^{-1 / d}=\rho^{-1 / d}$ ), and then, since each block contains one single target, the detection probability to find any of the targets in the whole domain can be approximated as the detection probability to find a single target in a single block with periodic boundary conditions. Then one reduces (3) to (1), where now the functions $q$ and $f$ are defined in just one of these single blocks.

Expressions (1) and (2), with $N>1$, are then strictly valid only for regularly spaced targets. Note that they also hold exactly for the two-target case $N=2$ with any target

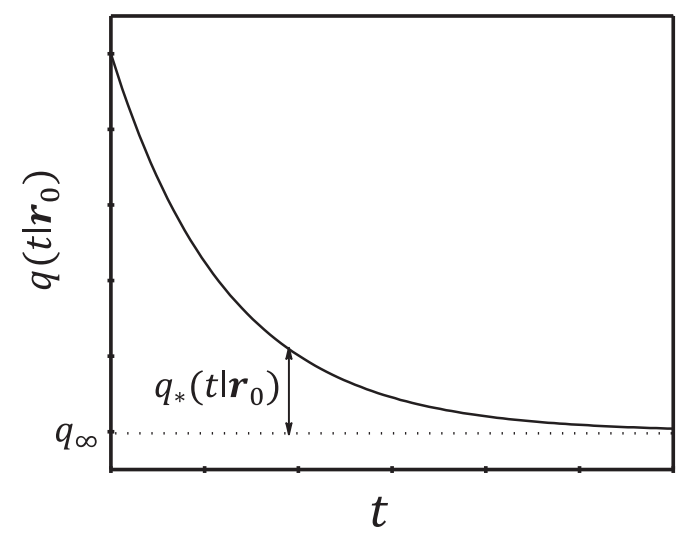

FIG. 2. Schematic definition of the asymptotic state $q_{\infty}$ so that $q\left(t \mid \mathbf{r}_{0}\right) \equiv q_{\infty}+q_{*}\left(t \mid \mathbf{r}_{0}\right)$.

distribution due to the symmetry of the corresponding problem. However, here we show that the same argument can still be used as a homogenization approximation for more complicated situations, for example, for Poisson distributed targets, provided that the mean distance between targets is large enough. In that case one can still assume that the division of the domain into blocks with one single target within holds on average. This will be confirmed below through comparison with Monte Carlo simulations.

Provided (2) and (3) hold, the mean value of the firstdetection distribution is computed through

$$
\begin{aligned}
\langle T\rangle & \equiv \int_{0}^{\infty} t f\left(t \mid \mathbf{r}_{0}\right) d t=-\lim _{s \rightarrow 0} \frac{d f\left(s \mid \mathbf{r}_{0}\right)}{d s} \\
& =\frac{q_{*}\left(\mathbf{r}_{\text {targ }}\right)-q_{*}\left(\mathbf{r}_{0}\right)}{q_{\infty}}+\frac{1}{q_{\infty}}
\end{aligned}
$$

where in the last identity we have used (2) and $q\left(t \mid \mathbf{r}_{0}\right) \equiv q_{\infty}+$ $q_{*}\left(t \mid \mathbf{r}_{0}\right)$. The meaning of this decomposition for the detection probability is illustrated in Fig. 2; when the motion process followed by the searcher within the finite domain reaches the stationary state, then the detection probability will tend to a constant value we term $q_{\infty}$. Provided the convergence of the function to the stationary state is fast enough, then the negative integral $\int_{0}^{\infty} q_{*}\left(t \mid \mathbf{r}_{0}\right) d t$ will be finite, which means that

$$
\lim _{s \rightarrow 0} q\left(s \mid \mathbf{r}_{0}\right)=\frac{q_{\infty}}{s}+\lim _{s \rightarrow 0} q_{*}\left(s \mid \mathbf{r}_{0}\right) \equiv \frac{q_{\infty}}{s}+q_{*}\left(\mathbf{r}_{0}\right)
$$

holds in the Laplace space, with $q_{*}$ and $q_{\infty}$ independent of $s$.

While we have derived expression (5) in an intuitive manner for the sake of clarity, we note that a more rigorous treatment could be done following the lines by Condamin and coworkers (see, e.g., $[8,28]$ and references therein). In that case one could interpret $q\left(t \mid \mathbf{r}_{0}\right)$ as the Green's function for the motion-plusdetection process followed by the searcher and $q_{*}\left(t \mid \mathbf{r}_{0}\right)$ as the corresponding pseudo-Green's function [29]. Therefore, by virtue of the renewal approximation introduced in (1) (which would be strictly valid if we consider a Markovian motion process) one can see that the formal structure of our result (4) coincides with that for first-passage problems analyzed before along these arguments [8]. 
Now, from the MDT expression (4) one can identify for convenience

$$
\left\langle T_{1}\right\rangle \equiv \frac{q_{*}\left(\mathbf{r}_{\text {targ }}\right)-q_{*}\left(\mathbf{r}_{0}\right)}{q_{\infty}}, \quad\left\langle T_{2}\right\rangle \equiv \frac{1}{q_{\infty}},
$$

so $\langle T\rangle=\left\langle T_{1}\right\rangle+\left\langle T_{2}\right\rangle$. Note that the first of the two contributions to the MDT (termed $\left\langle T_{1}\right\rangle$ ) vanishes for $\mathbf{r}_{0}=\mathbf{r}_{\text {targ }}$; this means that the second contribution $\left\langle T_{2}\right\rangle$ represents the MDT for a search that starts at a point arbitrarily close to the target. Hence, $\left\langle T_{1}\right\rangle$ can be properly interpreted as the initial time required to reach the target position starting from arbitrary $\mathbf{r}_{0}$. Actually, the latter can be properly interpreted by virtue of Kac's recurrence theorem [30], which states that the average return or recurrence time for any stationary stochastic process will equal the inverse of the actual probability of seeing the event. Basically, our renewal approach leads indirectly to the approximation $\left\langle T_{1}+T_{2}\right\rangle=\left\langle T_{1}\right\rangle+\left\langle T_{2}\right\rangle$, where $T_{1}$ corresponds to the approximated first-passage time (i.e., the time to reach the target) and $T_{2}$ is the return time conditioned to the specific detection mechanism considered, which takes into account the possibility of multiple revisits to the target position before detection is successful.

Below we shall confirm that this interpretation in terms of $T_{1}$ and $T_{2}$ is meaningful by verifying how both average times scale with the characteristic parameters $\rho, L$, and $a$ and by showing direct comparison to Monte Carlo simulations.

\section{EXAMPLES}

To compute the exact expressions for $q_{\infty}$ and $q_{*}$ appearing in (4) one needs to specify the motion pattern used by the searcher and the detection constraints involved. To illustrate how this works in practice, we consider the searcher a random walker performing consecutive flights at random speed $v$ and duration $\tau$ (Fig. 1), whose statistics are governed by arbitrary probability distributions of flight times $\varphi(t)$ and flight speeds $h(v)$ (note that Brownian and persistent motions represent limit cases of this general model [31,32]). Accordingly, we use $\left\langle t^{n}\right\rangle$ and $\left\langle v^{n}\right\rangle$ to denote the $n$ th-order moment of these distributions, respectively, and $l_{f} \equiv \sqrt{\left\langle v^{2}\right\rangle\left\langle t^{2}\right\rangle}$ represents the characteristic distance covered in a single flight. For systems with two or higher dimensions we will consider for simplicity that reorientations (i.e., changes in the direction of motion after each single flight) follow a uniform distribution (while this assumption could be relaxed, we do not explore here that possibility for the sake of simplicity). Finally, we introduce for convenience $p\left(\mathbf{r}, v, t \mid \mathbf{r}_{0}\right)$ as the probability density that the searcher is at position $\mathbf{r}$ at time $t$ moving with speed $v$ and $j\left(\mathbf{r}, t \mid \mathbf{r}_{0}\right)$ is the probability density that a single flight finishes at position $\mathbf{r}$ at time $t$.

Given the random-walk framework described, we have that the probability that a single flight covers a trajectory $\mathbf{r}$ at a time $t$ is

$$
\Psi(\mathbf{r}, t)=\int_{0}^{\infty} \delta\left(\mathbf{r}-v t \mathbf{u}_{\theta}\right) h(v) \varphi(t) d v
$$

where $\delta(\cdot)$ is the Dirac delta function and $\mathbf{u}_{\theta}$ is a unit vector in the direction of motion. Similarly, the probability that a single flight has not finished yet after a time $t$ (during which a trajectory $\mathbf{r}$ has been covered) will be

$$
\phi(\mathbf{r}, t)=\int_{0}^{\infty} d v \delta\left(\mathbf{r}-v t \mathbf{u}_{\theta}\right) h(v) \int_{t}^{\infty} d t^{\prime} \varphi\left(t^{\prime}\right) .
$$

From this, we can now use the typical prescriptions of continuous-time random walks [32-34] to write balance equations for the two probability densities $p\left(\mathbf{r}, v, t \mid \mathbf{r}_{0}\right)$ and $j\left(\mathbf{r}, t \mid \mathbf{r}_{0}\right)$ in the form

$$
\begin{aligned}
j\left(\mathbf{r}, t \mid \mathbf{r}_{0}\right)= & \int_{0}^{t} d t^{\prime} \int_{-\infty}^{\infty} d \mathbf{r}^{\prime} j\left(\mathbf{r}-\mathbf{r}^{\prime}, t-t^{\prime} \mid \mathbf{r}_{0}\right) \Psi\left(\mathbf{r}^{\prime}, t^{\prime}\right) \\
& +j_{0}(\mathbf{r}) \delta(t), \\
p\left(\mathbf{r}, v, t \mid \mathbf{r}_{0}\right)= & h(v) \int_{0}^{t} d t^{\prime} \int_{-\infty}^{\infty} d \mathbf{r}^{\prime} j\left(\mathbf{r}-\mathbf{r}^{\prime}, t-t^{\prime} \mid \mathbf{r}_{0}\right) \phi\left(\mathbf{r}^{\prime}, t^{\prime}\right),
\end{aligned}
$$

where $j_{0}(\mathbf{r})$ stands for the initial condition.

Now, in order to find closed expressions for $p$ and $j$ it is convenient again to transform (9) and (10) by Laplace; by doing that we obtain

$$
\begin{gathered}
j\left(\mathbf{r}, s \mid \mathbf{r}_{0}\right)=\int_{-\infty}^{\infty} j\left(\mathbf{r}-\mathbf{r}^{\prime}, s \mid \mathbf{r}_{0}\right) \Psi\left(\mathbf{r}^{\prime}, s\right) d \mathbf{r}^{\prime}+j_{0}(\mathbf{r}), \\
p\left(\mathbf{r}, v, s \mid \mathbf{r}_{0}\right)=h(v) \int_{-\infty}^{\infty} j\left(\mathbf{r}-\mathbf{r}^{\prime}, s \mid \mathbf{r}_{0}\right) \phi\left(\mathbf{r}^{\prime}, s\right) d \mathbf{r}^{\prime} .
\end{gathered}
$$

Note that $j$ and $p$ are originally defined in an infinite spatial domain. In order to adapt this formalism to a bounded domain and to introduce adequate boundary conditions one needs to use periodic summation techniques [22,35]. This leads to an extension of (11) and (12) which looks formally the same as that system of equations, but now $j$ and $p$ must be seen as periodic functions in space whose expression within the finite domain of volume $L^{d}$ coincides with the solution we are looking for. Therefore, one has to consider explicitly $j_{0}(\mathbf{r})$ as a periodic initial condition; for instance, if the initial condition of the searcher is $\mathbf{r}_{0}$, then the extension to the periodic case requires

$$
j_{0}(\mathbf{r})=\sum_{m_{1}=-\infty}^{\infty} \ldots \sum_{m_{d}=-\infty}^{\infty} \delta\left(\mathbf{r}-\mathbf{r}_{0}+L \mathbf{m}\right),
$$

with $\mathbf{m} \equiv\left(m_{1}, \ldots, m_{d}\right)$. We can benefit now from the periodic nature of $j$ and $p$ to propose a $d$-dimensional Fourier series expansion,

$$
\begin{aligned}
& j\left(\mathbf{r}, t \mid \mathbf{r}_{0}\right)=\sum_{m_{1}=-\infty}^{\infty} \ldots \sum_{m_{d}=-\infty}^{\infty} b_{m_{1}, \ldots, m_{d}}\left(t, \mathbf{r}_{0}\right) e^{-2 i \pi \mathbf{m} \cdot \mathbf{r} / L}, \\
& p\left(\mathbf{r}, v, t \mid \mathbf{r}_{0}\right)=\sum_{m_{1}=-\infty}^{\infty} \ldots \sum_{m_{d}=-\infty}^{\infty} c_{m_{1}, \ldots, m_{d}}\left(v, t, \mathbf{r}_{0}\right) e^{-2 i \pi \mathbf{m} \cdot \mathbf{r} / L} .
\end{aligned}
$$

Introducing this formal solution into (11) and (12), one obtains

$$
\begin{gathered}
b_{m_{1}, \ldots, m_{d}}\left(t, \mathbf{r}_{0}\right)=\frac{e^{2 i \pi \mathbf{m} \cdot \mathbf{r}_{0} / L}}{L^{d}} \frac{1}{1-\hat{\Psi}\left(\frac{2 \mathbf{m} \pi}{L}, s\right)}, \\
c_{m_{1}, \ldots, m_{d}}\left(v, t, \mathbf{r}_{0}\right)=b_{m_{1}, \ldots, m_{d}}\left(t, \mathbf{r}_{0}\right) h(v) \hat{\phi}\left(\frac{2 \mathbf{m} \pi}{L}, s\right),
\end{gathered}
$$


where the hats denote Fourier transform in the spatial coordinates and the initial condition (13) has been used. We have then derived a formal solution for the density probabilities $j$ and $p$, so after introducing a specific detection mechanism, we will be able to compute the detection probability $q$, as we show in the next sections.

Before going to more specific examples, however, let us mention that the Fourier coefficients in (16) and (17) simplify in the asymptotic limit $L \rightarrow \infty$ to (see the Appendix)

$$
\begin{aligned}
& b_{m_{1}, \ldots, m_{d}}\left(t, \mathbf{r}_{0}\right)=\frac{e^{2 i \pi \mathbf{m} \cdot \mathbf{r}_{0} / L}}{L^{d}} \frac{1}{s\langle t\rangle+\frac{2 \pi^{2}\left\langle v^{2}\right\rangle\left\langle t^{2}\right\rangle \mathbf{m}^{2}}{d L^{2}}}, \\
& c_{m_{1}, \ldots, m_{d}}\left(v, t, \mathbf{r}_{0}\right)=\frac{e^{2 i \pi \mathbf{m} \cdot \mathbf{r}_{0} / L}}{L^{d}} \frac{h(v)\langle t\rangle}{s\langle t\rangle+\frac{2 \pi^{2}\left\langle v^{2}\right\rangle\left\langle t^{2}\right\rangle \mathbf{m}^{2}}{d L^{2}}} .
\end{aligned}
$$

Note that from (19), together with (15), one easily verifies that the stationary solution of the propagator is then

$$
p_{\infty}=\lim _{t \rightarrow \infty} p\left(\mathbf{r}, v, t \mid \mathbf{r}_{0}\right)=s c_{0, \ldots, 0}\left(v, t, \mathbf{r}_{0}\right)=\frac{h(v)}{L^{d}} .
$$

\section{A. Speed-selective mechanism}

First, we assume that the main constraint to detection comes from the searcher speed. We consider that whenever the searcher passes at speed $v$ over a point whose distance to the target is less than $a$, detection can occur with probability $\Omega(v)$ (so for most situations $\Omega(v)$ is expected to be a monotonically decreasing function, especially if searchers are living organisms [18]). This mechanism involves two different limit situations (Fig. 3): if the typical flight distance $l_{f}$ is much larger than $a$, then the target detection problem resembles that of particle scattering, so the searcher effectively sees the target as a cross section of radius a (cross-limiting detection). Instead, for $l_{f}$ smaller than $a$ the detection typically occurs through (or close to) the surface of an imaginary surface of radius $a$ around the target (surface-limiting detection). The corresponding expression for the detection probability within

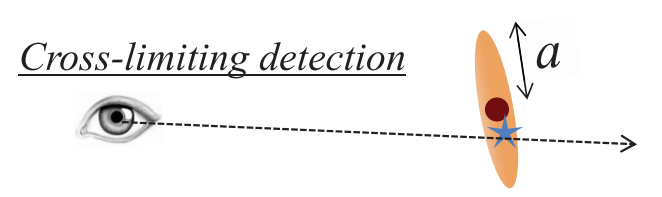

\section{$\underline{\text { Surface-limiting detection }}$}
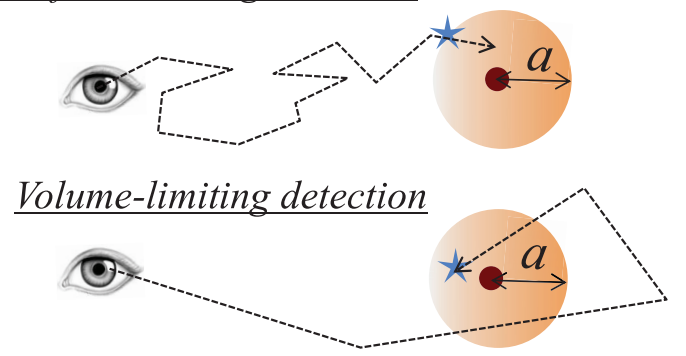

FIG. 3. (Color online) Schematic description of the cases of cross-limiting, surface-limiting, and volume-limiting detection. The dotted line represents the searcher trajectory, and the star is used to indicate the point at which detection will presumably occur. the interval $(t, t+d t)$ reads [22,36]

$$
\begin{aligned}
q\left(t \mid \mathbf{r}_{0}\right) d t & =\int_{0}^{\infty} d v \Omega(v) \int_{S} d S \int_{a-v_{r} d t}^{a} p\left(\mathbf{r}, v, t \mid \mathbf{r}_{0}\right) \\
& \approx \int_{0}^{\infty} d v \Omega(v) \frac{v}{2} \int_{S} p\left(\mathbf{r}, v, t \mid \mathbf{r}_{0}\right) d S d t
\end{aligned}
$$

Here, $S$ represents the surface (of radius $a$ ) over which the detection process takes place (the cross section or the effective target surface, depending on each specific situation). In the approximation, we have used first-order expansion on $d t$, while the factor $1 / 2$ on the speed comes from averaging the radial component of the speed $v_{r}$ over all possible angles of incidence on the surface $S$.

Introducing now the Fourier expansion (15) and rewriting conveniently the domain size in terms of the target density $\left(L \rightarrow \rho^{-1 / d}\right)$, one has

$$
\begin{aligned}
q\left(t \mid \mathbf{r}_{0}\right)= & \sum_{m_{1}=-\infty}^{\infty} \ldots \sum_{m_{d}=-\infty}^{\infty} \int_{0}^{\infty} d v \Omega(v) \frac{v}{2} \int_{S} c_{m_{1}, \ldots, m_{d}}\left(v, t, \mathbf{r}_{0}\right) \\
& \times e^{-2 i \pi \mathbf{m} \cdot\left(\mathbf{r}_{\text {targ }}+a \mathbf{u}_{r}\right) \rho^{1 / d}} d S,
\end{aligned}
$$

where $\mathbf{r}_{\text {targ }}+a \mathbf{u}_{r}$ represents the position vector of the points where the target detection could presumably occur, with $\mathbf{r}_{\text {targ }}$ being the target position and $\mathbf{u}_{r}$ being a unit vector in the radial direction. Now, we find the stationary detection probability, which, joining (20) and (21), is

$q_{\infty}=\int_{0}^{\infty} d v \Omega(v) v \int_{S} p_{\infty} d S=\rho S \int_{0}^{\infty} \frac{v}{2} h(v) \Omega(v) d v$,

or, equivalently,

$$
\left\langle T_{2}\right\rangle \equiv \frac{1}{q_{\infty}}=\frac{1}{\rho} \frac{2}{S \int_{0}^{\infty} v h(v) \Omega(v) d v} .
$$

Next, we calculate $q_{*}\left(\mathbf{r}_{0}\right)$ through its definition (5):

$$
\begin{aligned}
q_{*}\left(\mathbf{r}_{0}\right)= & \lim _{s \rightarrow 0} q\left(s \mid \mathbf{r}_{0}\right)-\frac{q_{\infty}}{s}=\lim _{s \rightarrow 0} \sum_{\mathbf{m} \neq 0} \int_{0}^{\infty} d v \Omega(v) \\
& \times \frac{v}{2} \int_{S} c_{m_{1}, \ldots, m_{d}}\left(v, t, \mathbf{r}_{0}\right) e^{-2 i \pi \mathbf{m} \cdot\left(\mathbf{r}_{\text {targ }}+a \mathbf{u}_{r}\right) \rho^{1 / d}} d S \\
= & \rho^{1-2 / d} \frac{d\langle t\rangle}{2 \pi^{2}\left\langle v^{2}\right\rangle\left\langle t^{2}\right\rangle} \sum_{\mathbf{m} \neq 0} \frac{1}{\mathbf{m}^{2}} \int_{0}^{\infty} d v \Omega(v) h(v) \\
& \times \frac{v}{2} \int_{S} e^{2 i \pi \mathbf{m} \cdot\left(\mathbf{r}_{0}-\mathbf{r}_{\text {targ }}\right) \rho^{1 / d}} e^{-2 i \pi a \mathbf{m} \cdot \mathbf{u}_{r} \rho^{1 / d}} d S,
\end{aligned}
$$

where $\sum_{\mathbf{m} \neq 0}$ denotes the sum over all possible values of $\left(m_{1}, m_{2}, \ldots\right)$ except for $m_{1}=m_{2}=\cdots=0$. So we observe in the previous expression that for $\mathbf{r}_{0}=\mathbf{r}_{\text {targ }}$ the term in the integral simplifies, while for an initial condition distributed uniformly throughout the domain it is easy to verify that $q_{*}\left(\mathbf{r}_{0}\right) \rightarrow 0$. This leads immediately to

$$
\begin{aligned}
\left\langle T_{1}\right\rangle & \equiv \frac{q_{*}\left(\mathbf{r}_{t a r g}\right)-q_{*}\left(\mathbf{r}_{0}\right)}{q_{\infty}} \\
& =\frac{1}{\rho^{2 / d}} \frac{d\langle t\rangle}{2 \pi^{2} l_{f}^{2}} \sum_{\mathbf{m} \neq 0} \frac{1}{S \mathbf{m}^{2}} \int_{S} e^{-2 i \pi a \mathbf{m} \cdot \mathbf{u}_{\mathbf{r}} \rho^{1 / d}} d S,
\end{aligned}
$$

where (23) has been used, as well as $l_{f}^{2} \equiv\left\langle v^{2}\right\rangle\left\langle t^{2}\right\rangle$. Note that the integral in the previous expression can be calculated exactly 


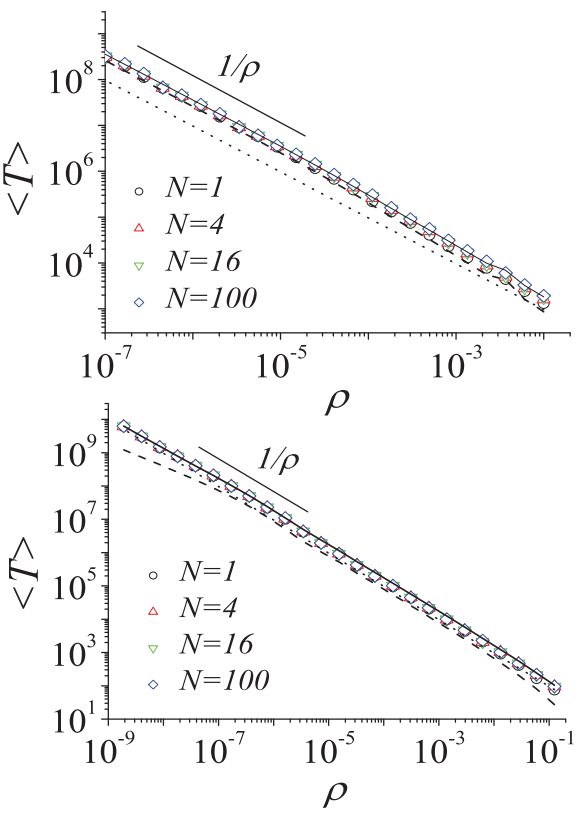

FIG. 4. (Color online) MDT for the SSM in (top) two and (bottom) three dimensions with $l_{f}=0.5 a$. We show the results for the MDT (solid line) compared to Monte Carlo simulations (symbols) obtained after averaging over $10^{4}$ realizations of the random walk process. The separate analytical values for $\left\langle T_{1}\right\rangle$ (dashed lines) and $\left\langle T_{2}\right\rangle$ (dotted lines) are also presented for a complete comparison. Different values for the number of homogeneously distributed targets $N$ have been used (see legends) while keeping the target density constant to reach a proper comparison. In all cases we have chosen $a=0.5, \varphi(t)=\delta(t-1), h(v)=\frac{1}{\langle v\rangle} e^{-v /\langle v\rangle}$, and $\Omega(v)=\Theta(\langle v\rangle-v)$.

provided we specify the dimensionality of the system $d$ and the specific surface $S$. For example, in two and three dimensions for the surface-limiting case we get, respectively,

$$
\begin{gathered}
\left\langle T_{1}\right\rangle=\frac{1}{\rho} \frac{\langle t\rangle}{\pi^{2} l_{f}^{2}} \sum_{\mathbf{m} \neq 0} \frac{J_{0}\left(|\mathbf{m}| a \pi \rho^{1 / d}\right)}{\mathbf{m}^{2}}, \\
\left\langle T_{1}\right\rangle=\frac{1}{\rho^{2 / 3}} \frac{3\langle t\rangle}{2 \pi^{2} l_{f}^{2}} \sum_{\mathbf{m} \neq 0} \frac{\sin \left(|\mathbf{m}| a \pi \rho^{1 / d}\right)}{a \pi \mathbf{m}^{3}},
\end{gathered}
$$

where $J_{0}$ represents the zero order of the Bessel function of the first kind.

Similarly, for the cross-limiting case a first-order expansion in $\rho^{-1}$ leads to

$$
\begin{gathered}
\left\langle T_{1}\right\rangle=\frac{1}{\rho} \frac{\langle t\rangle}{\pi^{2} l_{f}^{2}} \sum_{\mathbf{m} \neq 0} \frac{1}{\mathbf{m}^{2}} \\
\left\langle T_{1}\right\rangle=\frac{1}{\rho^{2 / 3}} \frac{3\langle t\rangle}{\pi^{2} l_{f}^{2}} \sum_{\mathbf{m} \neq 0} \frac{1}{\mathbf{m}^{2}}
\end{gathered}
$$

for two and three dimensions, respectively. Therefore, putting together these results with (24) one obtains the corresponding analytical expressions for the $\operatorname{MDT}\langle T\rangle=\left\langle T_{1}\right\rangle+\left\langle T_{2}\right\rangle$.

The validity of our approach is finally confirmed by comparing our theoretical expressions with Monte Carlo simulations. We provide results for both $a>l_{f}$ (Fig. 4), which corresponds to the surface-limiting case, and $a<l_{f}$ (cross-limiting case, Fig. 5) and also for very different numbers of targets $N$
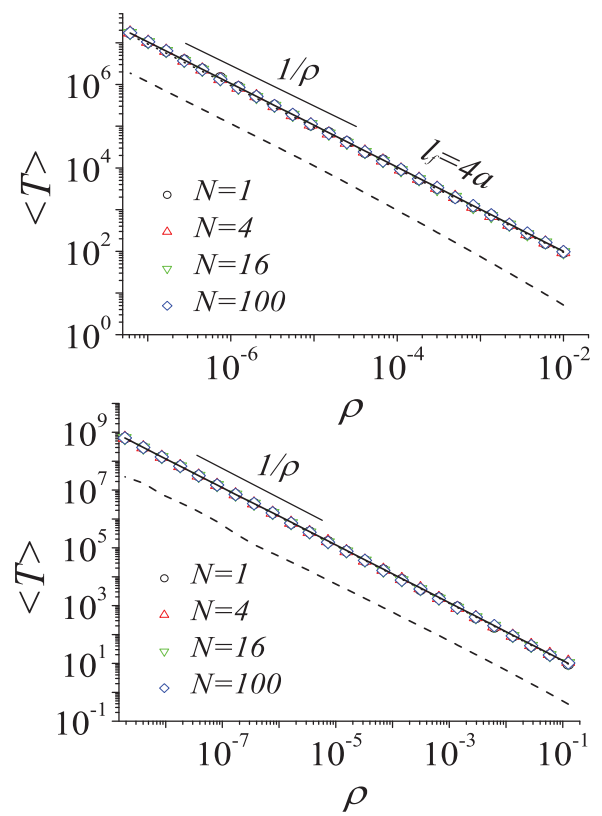

FIG. 5. (Color online) MDT for the SSM in (top) two and (bottom) three dimensions with $l_{f}=4 a$. We show the results for the MDT (solid line) compared to Monte Carlo simulations (symbols) obtained after averaging over $10^{4}$ realizations of the random walk process. The separate analytical values for $\left\langle T_{1}\right\rangle$ (dashed lines) and $\left\langle T_{2}\right\rangle$ (dotted lines) are also presented for a complete comparison. Different values for the number of homogeneously distributed targets $N$ have been used (see legends) while keeping the target density constant to reach a proper comparison. In all cases we have chosen $a=0.5, \varphi(t)=\delta(t-1), h(v)=\frac{1}{\langle v\rangle} e^{-v /\langle v\rangle}$, and $\Omega(v)=\Theta(\langle v\rangle-v)$.

(while keeping the target density $N / L^{d}$ constant) to verify that the deviations from the case $N=1$ are minimal. Poisson distributions have been used in all the cases to determine the targets positions; that has been done to verify that even under conditions where target distributions are not strictly regular in space, our approach provides a good approximation to the MDT.

In Fig. 4 one sees that in both two and three dimensions the agreement found between our analytical prediction for the MDT and the results from simulations is excellent. For the sake of completeness, we show in the same plots the analytical predictions for both $\left\langle T_{1}\right\rangle$ (dashed lines) and $\left\langle T_{2}\right\rangle$ (dotted lines). Note that the scaling of $\langle T\rangle$ with $\rho$ can vary from two to three dimensions, depending on the term that dominates (either $\left\langle T_{1}\right\rangle$ or $\left\langle T_{2}\right\rangle$ ). In two dimensions both times scale as $\rho^{-1}$, so that is the scaling found as long as the target density becomes small, so the asymptotic regime is reached. In three dimensions, however, we have $\left\langle T_{1}\right\rangle \sim \rho^{-2 / 3}$ and $\left\langle T_{2}\right\rangle \sim \rho^{-1}$. For low enough $\rho$ the return time $\left\langle T_{2}\right\rangle$ is expected to dominate; however, we have checked that the transitory regime spans several orders in which both $\left\langle T_{1}\right\rangle$ and $\left\langle T_{2}\right\rangle$ are of the same order of magnitude (at least for the parameters used in our simulations). Anyway, it is found that in this transient regime $\left\langle T_{1}\right\rangle$ scales also like $\rho^{-1}$, so that is the scaling found for the overall range (but note how the regime $\left\langle T_{1}\right\rangle \sim \rho^{-2 / 3}$ is finally reached for target densities below $10^{-7}$ in the plot).

Very similar arguments hold for the cross-limiting case in Fig. 5. The only remarkable difference with the previous 
case is that now since a larger flight distance $l_{f}$ has been used (without changing the other parameters), the time $\left\langle T_{1}\right\rangle$ necessary to reach the target decreases. Therefore, one observes correspondingly that the MDT is clearly dominated by the return time $\left\langle T_{2}\right\rangle$.

\section{B. Stop-and-scan mechanism}

Now, we will consider that detection can occur with probability $\Phi(r)$ any time the searcher finishes a single flight at a distance $r$ from the target location, where $\Phi(r)$ should decay rapidly for large $r$. This reflects the possibility that the searcher cannot detect its target while moving and needs to stop and scan its surrounding media, in analogy to the idea of intermittent searches broadly explored in recent years [37-39]), also with partial detection abilities [40]. The effective detection size could then be conveniently defined as $a \equiv A \int_{0}^{\infty} r \Phi(r) d r$, with $A^{-1}=\int_{0}^{\infty} \Phi(r) d r$ being a normalization factor. The detection probability for this case reads then, by definition,

$$
q\left(t \mid \mathbf{r}_{0}\right)=\int_{V} \Phi(r) j\left(\mathbf{r}, t \mid \mathbf{r}_{0}\right) d V,
$$

where $V$ represents the effective volume over which detection is possible (we term this situation volume-limiting detection; see Fig. 1).

Following the same derivation as in the previous section, we could write this expression using a Fourier series as

$$
\begin{aligned}
q\left(t \mid \mathbf{r}_{0}\right)= & \sum_{m_{1}=-\infty}^{\infty} \ldots \sum_{m_{d}=-\infty}^{\infty} \int_{V} b_{m_{1}, \ldots, m_{d}}\left(t, \mathbf{r}_{0}\right) \\
& \times e^{-2 i \pi \mathbf{m} \cdot\left(\mathbf{r}_{\text {targ }}+r \mathbf{u}_{r}\right) \rho^{1 / d}} d V,
\end{aligned}
$$

which leads to the stationary solution

$$
q_{\infty}=\int_{V} b_{0, \ldots, 0}\left(t, \mathbf{r}_{0}\right) d V=\rho\langle t\rangle \int_{V} \Phi(r) d V .
$$

From here one infers

$$
\left\langle T_{2}\right\rangle=\frac{1}{\rho} \frac{1}{\langle t\rangle \int_{V} \Phi(r) d V} .
$$

On the other hand, to compute the term $\left\langle T_{1}\right\rangle$ we proceed as follows:

$$
\begin{aligned}
q_{*}\left(\mathbf{r}_{0}\right)= & \lim _{s \rightarrow 0} q\left(s \mid \mathbf{r}_{0}\right)-\frac{q_{\infty}}{s} \\
= & \lim _{s \rightarrow 0} \sum_{\mathbf{m} \neq 0} \int_{V} \Phi(r) b_{m_{1}, \ldots, m_{d}}\left(s, \mathbf{r}_{0}\right) e^{-2 i \pi \mathbf{m} \cdot\left(\mathbf{r}_{\text {targ }}+r \mathbf{u}_{r}\right) \rho^{1 / d}} d V \\
= & \rho^{1-2 / d} \frac{d\langle t\rangle}{2 \pi^{2}\left\langle v^{2}\right\rangle\left\langle t^{2}\right\rangle} \sum_{\mathbf{m} \neq 0} \frac{1}{\mathbf{m}^{2}} \int_{V} \Phi(r) e^{2 i \pi \mathbf{m} \cdot\left(\mathbf{r}_{0}-\mathbf{r}_{\text {targ }}\right) \rho^{1 / d}} \\
& \times e^{-2 i \pi r \mathbf{m} \cdot \mathbf{u}_{r} \rho^{1 / d}} d V .
\end{aligned}
$$

Again, if we consider $\mathbf{r}_{0}$ to be uniformly distributed one obtains $q_{*}\left(\mathbf{r}_{0}\right) \rightarrow 0$, and as a consequence,

$$
\begin{aligned}
\left\langle T_{1}\right\rangle & =\frac{q_{*}\left(\mathbf{r}_{\text {targ }}\right)-q_{*}\left(\mathbf{r}_{0}\right)}{q_{\infty}} \\
& =\frac{1}{\rho^{2 / d}} \frac{d\langle t\rangle}{2 \pi^{2} l_{f}^{2}} \sum_{\mathbf{m} \neq 0} \frac{1}{\mathbf{m}^{2}}\left[\frac{\int_{V} \Phi(r) e^{-2 i \pi r \mathbf{m} \cdot \mathbf{u}_{r} \rho^{1 / d} d V}}{\int_{V} \Phi(r) d V}\right] .
\end{aligned}
$$
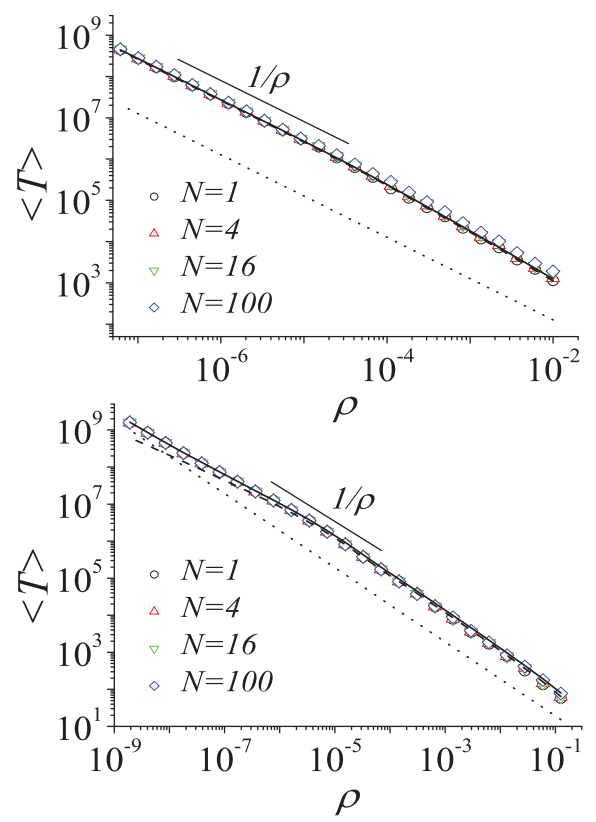

FIG. 6. (Color online) MDT for the SaSM in (top) two and (bottom) three dimensions with $l_{f}=0.5 a$. We show the results for the MDT (solid line) compared to Monte Carlo simulations (symbols) obtained after averaging over $10^{4}$ realizations of the random walk process. The separate analytical values for $\left\langle T_{1}\right\rangle$ (dashed lines) and $\left\langle T_{2}\right\rangle$ (dotted lines) are also presented for a complete comparison. Different values for the number of homogeneously distributed targets $N$ have been used (see legends) while keeping the target density constant to reach a proper comparison. In all cases we have chosen $a=0.5, \varphi(t)=\delta(t-1), h(v)=\frac{1}{\langle v\rangle} e^{-v /\langle v\rangle}$, and $\Phi(r)=\Theta(a-r)$.

The two- and three-dimensional cases can be exactly computed also for the stop-and-scan mechanism (SaSM); they will read, respectively,

$$
\begin{gathered}
\left\langle T_{1}\right\rangle=\frac{1}{\rho} \frac{\langle t\rangle}{\pi^{2} l_{f}^{2}} \sum_{\mathbf{m} \neq 0} \frac{\int_{0}^{\infty} r \Omega(r) J_{0}\left(|\mathbf{m}| r \pi \rho^{1 / d}\right) d r}{\mathbf{m}^{2} \int_{0}^{\infty} r \Omega(r) d r} \\
\left\langle T_{1}\right\rangle=\frac{1}{\rho^{2 / 3}} \frac{3\langle t\rangle}{2 \pi^{2} l_{f}^{2}} \sum_{\mathbf{m} \neq 0} \frac{\int_{0}^{\infty} r \Omega(r) \sin \left(|\mathbf{m}| r \pi \rho^{1 / d}\right) d r}{|\mathbf{m}|^{3} \int_{0}^{\infty} r^{2} \Omega(r) d r} .
\end{gathered}
$$

Note that the scaling found at the leading order $\left(\left\langle T_{1}\right\rangle \sim \rho^{-2 / d}\right.$ and $\left.\left\langle T_{2}\right\rangle \sim \rho^{-1}\right)$ coincides with that we found for the speed-selective mechanism (SSM) case above; in the next section we will show that this law can be generalized to a large class of detection mechanisms. If we now compare again the expressions obtained with Monte Carlo simulations [taking $\Omega(r)$ as a Heaviside function which decays from 1 to 0 at $r=a$ ], we find again really good agreement (Figs. 6 and 7). We have also distinguished here between the cases $l_{f}<a$ and $l_{f}>a$, although in the SaSM case the expression derived is exactly the same for both. However, the distinction has been made here to emphasize that for low values of $l_{f}$ the searcher needs a lot of time to reach the target for the first time, so in this situation $\left\langle T_{1}\right\rangle$ (represented by dashed lines) is expected to be dominant, as confirmed by Fig. 6. The contrary happens for larger $l_{f}$, so in Fig. 7 the dotted lines, which correspond to $\left\langle T_{2}\right\rangle$, dominate instead. Regarding the scaling of 

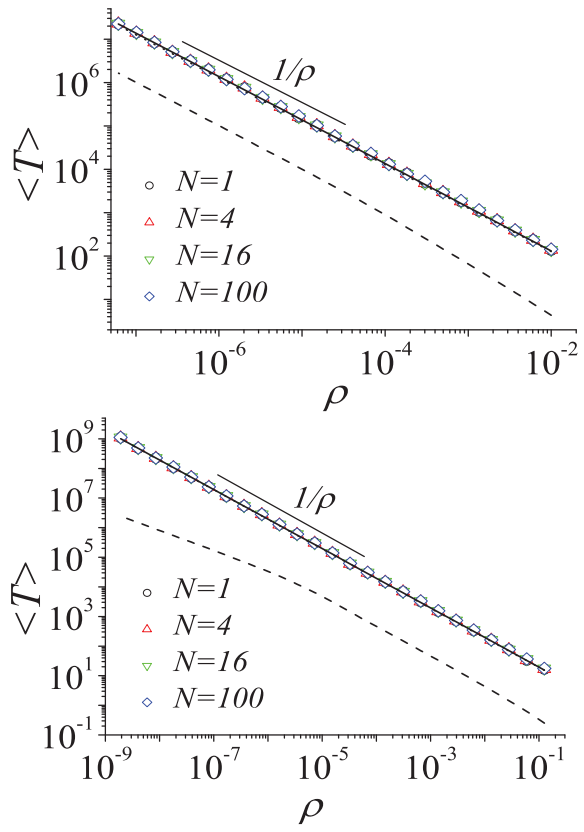

FIG. 7. (Color online) MDT for the SaSM in (top) two and (bottom) three dimensions with $l_{f}=0.5 a$. We show the results for the MDT (solid line) compared to Monte Carlo simulations (symbols) obtained after averaging over $10^{4}$ realizations of the random walk process. The separate analytical values for $\left\langle T_{1}\right\rangle$ (dashed lines) and $\left\langle T_{2}\right\rangle$ (dotted lines) are also presented for a complete comparison. Different values for the number of homogeneously distributed targets $N$ have been used (see legends) while keeping the target density constant to reach a proper comparison. In all cases we have chosen $a=0.5, \varphi(t)=\delta(t-1), h(v)=\frac{1}{\langle v\rangle} e^{-v /\langle v\rangle}$, and $\Phi(r)=\Theta(a-r)$.

the MDT with $\rho$, we observe exactly the same behavior as that for the SSM case. The scaling $\langle T\rangle \sim \rho^{-1}$ holds always in two dimensions, and the same happens in three dimensions except for a transient time for the case $l_{f}=0.5 a$ (approximately in the range $10^{-8}<\rho<10^{-5}$ ). There the dominance of $\left\langle T_{1}\right\rangle$ leads to $\langle T\rangle \sim \rho^{-2 / 3}$, as expected from (38). In the asymptotic regime, however, $\left\langle T_{2}\right\rangle \sim \rho^{-1 / d}$ will become dominant again (this cannot be well observed in Fig. 6 due to the enormous computational times it would require to reach smaller values of $\rho$ ).

\section{GENERAL SCALING OF THE MDT}

The two specific detection mechanisms explored in the previous section (SSM and SaSM) yield a common asymptotic scaling on the target density: $\left\langle T_{1}\right\rangle \sim \rho^{-2 / d}$ and $\left\langle T_{2}\right\rangle \sim \rho^{-1}$. In the following, we extend the derivations followed there to prove that this result holds for any detection mechanism where the detection probability can be written as

$$
q\left(t \mid \mathbf{r}_{0}\right)=\mathcal{G}\left[p\left(\mathbf{r}, v, t \mid \mathbf{r}_{0}\right)\right]+\mathcal{H}\left[j\left(\mathbf{r}, t \mid \mathbf{r}_{0}\right)\right],
$$

with $\mathcal{H}$ and $\mathcal{G}$ representing arbitrary linear integral operators on the spatial coordinates $\mathbf{r}$ and/or the speed $v$. To verify this, one just has to use again the Fourier expansion (14) and (15) together with (18) and (19) to obtain

$$
\begin{aligned}
\lim _{s \rightarrow 0} q\left(s \mid \mathbf{r}_{0}\right)= & \lim _{s \rightarrow 0} \sum_{m_{1}=-\infty}^{\infty} \ldots \sum_{m_{d}=-\infty}^{\infty} \rho e^{2 i \pi \mathbf{m} \cdot \mathbf{r}_{0} \rho^{1 / d}} \\
& \times \frac{\langle t\rangle}{s\langle t\rangle+\frac{2 \pi^{2}\left\langle v^{2}\right\rangle\left\langle t^{2}\right\rangle \mathbf{m}^{2}}{d \rho^{-2 / d}}} \mathcal{G}\left[h(v) e^{-2 i \pi \mathbf{m} \cdot \mathbf{r} \rho^{1 / d}}\right] \\
& +\sum_{m_{1}=-\infty}^{\infty} \ldots \sum_{m_{d}=-\infty}^{\infty} \rho e^{2 i \pi \mathbf{m} \cdot \mathbf{r}_{0} \rho^{1 / d}} \\
& \times \frac{1}{s\langle t\rangle+\frac{2 \pi^{2}\left\langle v^{2}\right\rangle\left\langle t^{2}\right\rangle \mathbf{m}^{2}}{2 d \rho^{-2 / d}}} \mathcal{H}\left[e^{-2 i \pi \mathbf{m} \cdot \mathbf{r} \rho^{1 / d}}\right]
\end{aligned}
$$

Again, the stationary behavior corresponds to the contribution of the term $m_{1}=\cdots=m_{d}=0$, so we find

$$
q_{\infty}=\rho\left(\mathcal{G}[h(v)]+\frac{1}{\langle t\rangle} \mathcal{H}[1]\right) \sim \rho,
$$

and the contribution from the other terms in the expansion leads to

$$
\begin{aligned}
q_{*}\left(\mathbf{r}_{0}\right)= & \lim _{s \rightarrow 0} q\left(s \mid \mathbf{r}_{0}\right)-\frac{q_{\infty}}{s}=\rho^{1-2 / d} \frac{d\langle t\rangle}{2 \pi^{2}\left\langle v^{2}\right\rangle\left\langle t^{2}\right\rangle} \\
& \times \sum_{\mathbf{m} \neq 0} \frac{e^{-2 i \pi \mathbf{m} \cdot\left(\mathbf{r}_{\text {targ }}-\mathbf{r}_{0}\right) \rho^{1 / d}}}{\mathbf{m}^{2}} \mathcal{G}\left[h(v) e^{-2 i \pi r \mathbf{m} \cdot \mathbf{u}_{r} \rho^{1 / d}}\right] \\
& +\rho^{1-2 / d} \frac{d}{2 \pi^{2}\left\langle v^{2}\right\rangle\left\langle t^{2}\right\rangle} \sum_{\mathbf{m} \neq 0} \frac{e^{-2 i \pi \mathbf{m} \cdot\left(\mathbf{r}_{\text {targ }}-\mathbf{r}_{0}\right) \rho^{1 / d}}}{\mathbf{m}^{2}} \\
& \times \mathcal{H}\left[e^{-2 i \pi r \mathbf{m} \cdot \mathbf{u}_{r} \rho^{1 / d}}\right]
\end{aligned}
$$

after introducing $\mathbf{r}=\mathbf{r}_{\text {targ }}+r \mathbf{u}_{r}$. Hence, the two contributions to the MDT are found to obey in the target low-density limit $\rho \rightarrow 0$

$$
\begin{aligned}
\left\langle T_{1}\right\rangle & \equiv \frac{q_{*}\left(\mathbf{r}_{\text {targ }}\right)-q_{*}\left(\mathbf{r}_{0}\right)}{q_{\infty}} \sim \rho^{-2 / d}, \\
\left\langle T_{2}\right\rangle & \equiv \frac{1}{q_{\infty}} \sim \rho^{-1} .
\end{aligned}
$$

Note that in the case of $\left\langle T_{1}\right\rangle$, the multiplicative factor in front of $\rho^{-2 / d}$ will be independent of the specific detection mechanism at leading order (as expected, since this is nothing but the time to approach the target). Furthermore, by taking the number of targets $N$ as a fixed parameter, this leads to an equivalent scaling in terms of the domain size $\left\langle T_{1}\right\rangle \sim L^{2}$ and $\left\langle T_{2}\right\rangle \sim L^{d}$. This scaling on $L$ allows an easier interpretation in physical terms: now we check that the time necessary to approach the target asymptotically corresponds to a diffusive scaling law $\left(\left\langle T_{1}\right\rangle \sim L^{2}\right.$ ), while the return time $\left\langle T_{2}\right\rangle$ is proportional to the volume domain, in agreement with the statement from Kac's recurrence theorem discussed above.

\section{CONCLUSIONS}

We have tried here to provide a general framework to explore analytically the first-detection time distribution and the corresponding MDT, which holds for arbitrary dimensions and arbitrary detection mechanisms. As discussed in Sec. II, the approach proposed is exact for Markovian patterns of motion with regularly spaced targets, but in this work we have checked 
its goodness for much more complicated situations, such as non-Markovian motion (e.g., the continuous-time random walk used in our example cases), and more complicated target distributions (we have focused here on Poisson distributions, but we claim that we expect the approach to provide reasonably good approximations provided the departure from regularly spaced conditions is not extreme).

Apart from providing explicit expressions for $f\left(t \mid \mathbf{r}_{0}\right)$, $\langle T\rangle$ and checking the agreement of the results obtained against Monte Carlo simulations, we have been able to obtain rather general conclusions by considering a class of detection mechanisms [Eq. (39)] which includes most situations one can find in search processes in nature (either at the molecular or biological level). To summarize, we have found that, at the leading order in $\rho \rightarrow 0,\left\langle T_{1}\right\rangle \sim C_{1} \rho^{-2 / d}$ holds, where $C_{1}$ is a constant whose specific value depends on the dimension of the system but not on the specific detection mechanism considered. This confirms that $T_{1}$ basically corresponds to the time needed to approach the target. Likewise, $\left\langle T_{2}\right\rangle$ is found to be inversely proportional to the target density, and its scaling on the detection size $a$ will be different in general depending on the specific mechanism considered (note that for the SSM $\left\langle T_{2}\right\rangle \sim S^{-1} \sim a^{1-d}$ holds, while for the SaSM $\left.\left\langle T_{2}\right\rangle \sim V^{-1} \sim a^{-d}\right)$. As a result, the peculiarities of each detection mechanism make it impossible to find a universal scaling on $a$, contrary to what happens with the scaling on $\rho$. Note also that the scaling on $\rho$ obtained here at leading order coincides with the results obtained for corresponding first-passage problems [8,14]. Actually, our approach could be reduced to a first-passage process just by choosing the appropriate detection mechanism.

Let us finally stress that an essential ingredient within all this discussion comes from the use of a velocity model in our study (Fig. 1) to describe the movement of the particles, so a persistence in motion is explicitly considered. Instead, for the limit case of pure Brownian walkers one should take into account that particles move with an arbitrarily large speed while keeping the diffusion coefficient $D$ of particles constant (in our notation, this limit corresponds to taking $\left\langle v^{2}\right\rangle \rightarrow \infty$ and $\langle t\rangle \rightarrow 0$ while keeping the product $D \sim\left\langle v^{2}\right\rangle\langle t\rangle$ constant). In this limit, both $q_{\infty}$ and $q_{*}$ will tend to $\infty$, while the quotient $q_{*} / q_{\infty}$ will be finite. Then $\left\langle T_{1}\right\rangle$ will be the only term contributing to the MDT, in agreement with the idea that a Brownian particle which gets arbitrarily close to a target boundary will detect it with certainty in a vanishing time [1]. Therefore, our work shows that the role of the detection failure upon encounter in a random search process, which is the main focus of the present work, is intimately related to the explicit consideration of a persistence (or a relaxation time) in particle motion. These results can provide helpful insights into models for particle kinetics and search processes related to animal or human activities. We conclude then by stressing that one should next study how the scaling laws and the results reported here get modified when multiple searchers are introduced, as a natural generalization of the present work in order to gain still more realism in these approaches.

\section{ACKNOWLEDGMENTS}

We wish to thank the anonymous reviewers for helpful. This research has been partially supported by Grants No. FIS 2012-32334 (V.M., D.C.) and No. SGR 2009-00164 (V.M., D.C.). F.B. acknowledges the Spanish ministry of Science and Innovation (Grants No. REF.2009-04133 and No. BFU2010-22337).

\section{APPENDIX: DERIVATION OF EXPRESSIONS (18) AND (19)}

We have introduced in (7) and (8) the functions $\Psi$ and $\phi$ from the general continuous-time random walk framework considered there. The Fourier-Laplace transform of these can be conveniently written as

$$
\begin{aligned}
\hat{\Psi}(\mathbf{k}, s)= & \int_{-\infty}^{\infty} d \mathbf{r} e^{-i \mathbf{k} \cdot \mathbf{r}} \int_{0}^{\infty} d t e^{-s t} \Psi(\mathbf{r}, t) \\
= & \int_{-\infty}^{\infty} d \mathbf{r} e^{-i \mathbf{k} \cdot \mathbf{r}} \int_{0}^{\infty} d t e^{-s t} \varphi(t) \int_{0}^{\infty} d v \delta\left(\mathbf{r}-v t \mathbf{u}_{\theta}\right) h(v) \\
= & \int_{0}^{\infty} d t e^{-s t} \varphi(t) \int_{0}^{\infty} d v e^{-i v t \mathbf{k} \cdot \mathbf{u}_{\theta}} h(v), \\
\hat{\phi}(\mathbf{k}, s)= & \int_{-\infty}^{\infty} d \mathbf{r} \int_{0}^{\infty} d t e^{-i \mathbf{k} \cdot \mathbf{r}} e^{-s t} \phi(\mathbf{r}, t) \\
= & \int_{-\infty}^{\infty} d \mathbf{r} \int_{0}^{\infty} d t \int_{0}^{\infty} d v e^{-i \mathbf{k} \cdot \mathbf{r}} e^{-s t} \delta\left(\mathbf{r}-v t \mathbf{u}_{\theta}\right) \\
& \times h(v) \int_{t}^{\infty} d t^{\prime} \varphi\left(t^{\prime}\right) \\
= & \int_{0}^{\infty} d t \int_{0}^{\infty} d v e^{-i v t \mathbf{k} \cdot \mathbf{u}_{\theta}} e^{-s t} h(v) \int_{t}^{\infty} d t^{\prime} \varphi\left(t^{\prime}\right), \quad(\mathrm{A} 2)
\end{aligned}
$$

so after performing the asymptotic limit for large time and space with isotropic motion, one is left with the expressions

$$
\begin{aligned}
\lim _{s, \mathbf{k} \rightarrow 0} \hat{\Psi}(\mathbf{k}, s)= & \int_{0}^{\infty} d t \int_{0}^{\infty} d v\left(1-i v t \mathbf{k} \cdot \mathbf{u}_{\theta}-\frac{v^{2} t^{2} \mathbf{k}^{2}}{2}+\ldots\right) \\
& \times(1-s t+\ldots) h(v) \varphi(t) \\
= & 1-s\langle t\rangle-\frac{\left\langle v^{2}\right\rangle\left\langle t^{2}\right\rangle}{2 d} \mathbf{k}^{2}+\ldots, \\
\lim _{s, \mathbf{k} \rightarrow 0} \hat{\phi}(\mathbf{k}, s)= & \int_{0}^{\infty} d t \int_{0}^{\infty} d v\left(1-i v t \mathbf{k} \cdot \mathbf{u}_{\theta}-\frac{v^{2} t^{2} \mathbf{k}^{2}}{2}+\ldots\right) \\
& \times(1-s t+\ldots) h(v) \int_{t}^{\infty} d t^{\prime} \varphi\left(t^{\prime}\right) \\
= & \langle t\rangle+\ldots
\end{aligned}
$$

As a consequence, using the general expression of the Fourier coefficients (16) and (17), the corresponding asymptotic limit will reduce to expressions (18) and (19).
[1] S. Redner, A Guide to First-Passage Processes (Cambridge University Press, Cambridge, 2001).

[2] M. F. Shlesinger, Nature (London) 450, 40 (2007).
[3] C. Loverdo, O. Bénichou, M. Moreau, and R. Voituriez, Nat. Phys. 4, 134 (2008).

[4] F. Bartumeus and J. Catalán, J. Phys. A 42, 434002 (2009). 
[5] V. Méndez, D. Campos, and F. Bartumeus, Stochastic Foundations in Movement Ecology (Springer, Berlin, 2013).

[6] O. Bénichou, M. Coppey, M. Moreau, P.-H. Suet, and R. Voituriez, Phys. Rev. Lett. 94, 198101 (2005).

[7] G. M. Viswanathan, S. Buldyrev, S. Havlin, M. G. E. da Luz, E. P. Raposo, and H. E. Stanley, Nature (London) 381, 413 (1996).

[8] S. Condamin, O. Bénichou, V. Tejedor, R. Voituriez, and J. Klafter, Nature (London) 450, 77 (2007).

[9] L. K. Gallos, C. Song, S. Havlin, and H. A. Makse, Proc. Natl. Acad. Sci. USA 104, 7746 (2007).

[10] C. Mejía-Monasterio, G. Oshanin, and G. Schehr, J. Stat. Mech. Theor. Exp. (2011) P06022.

[11] C. Calero, J. Faraudo, and M. Aguilella-Arzo, Phys. Rev. E 83, 021908 (2011).

[12] S. Condamin, O. Bénichou, and M. Moreau, Phys. Rev. Lett. 95, 260601 (2005).

[13] J. D. Noh and H. Rieger, Phys. Rev. Lett. 92, 118701 (2004).

[14] V. Tejedor, R. Voituriez, and O. Bénichou, Phys. Rev. Lett. 108, 088103 (2012).

[15] T. G. Mattos, C. Mejía-Monasterio, R. Metzler, and G. Oshanin, Phys. Rev. E 86, 031143 (2012).

[16] D. S. Grebenkov, J. Chem. Phys. 132, 034104 (2010).

[17] W. J. O’Brien, H. I. Browman, and B. I. Evans, Am. Sci. 78, 152 (1990).

[18] D. L. Kramer and R. L. McLaughlin, Am. Zool. 41, 137 (2001).

[19] B. O. Koopman, Search and Screening: General Principles with Historical Applications (Pergamon, New York, 1980).

[20] L. D. Stone, Theory of Optimal Search (Operations Research of America, Arlington, VA, 1989).

[21] A. M. Reynolds and F. Bartumeus, J. Theor. Biol. 260, 98 (2009).

[22] D. Campos, V. Méndez, and F. Bartumeus, Phys. Rev. Lett. 108, 028102 (2012).
[23] Z. Koza and H. Taitelbaum, Phys. Rev. E 57, 237 (1998).

[24] S. B. Yuste, J. J. Ruiz-Lorenzo, and K. Lindenberg, Phys. Rev. E 74, 046119 (2006).

[25] E. Abad, S. B. Yuste, and K. Lindenberg, Phys. Rev. E 86, 061120 (2012).

[26] R. L. Stratonovich, Topics in the Theory of Random Noise (Gordon and Breach, New York, 1967).

[27] N. G. van Kampen, Stochastic Processes in Physics and Chemistry, 3rd ed. (Elsevier, Amsterdam, 2007).

[28] S. Condamin, V. Tejedor, R. Voituriez, O. Bénichou, and J. Klafter, Proc. Natl. Acad. Sci. USA 105, 5675 (2008).

[29] G. Barton, Elements of Green's Functions and Propagation (Oxford University Press, Oxford, 1989).

[30] M. Kac, Bull. Am. Math. Soc. 53, 1002 (1947).

[31] I. Gómez-Portillo, D. Campos, and V. Méndez, J. Stat. Mech. Theor. Exp. (2011) P02033.

[32] V. Zaburdaev, M. Schmiedeberg, and H. Stark, Phys. Rev. E 78, 011119 (2008).

[33] R. Metzler and J. Klafter, Phys. Rep. 339, 1 (2000).

[34] G. Zumofen and J. Klafter, Phys. Rev. E 47, 851 (1993).

[35] I. Calvo, B. A. Carreras, R. Sánchez, and B. Ph. van Milligen, J. Phys. A 40, 13511 (2008).

[36] T. Verechtchaguina, I. M. Sokolov, and L. Schimansky-Geier, Phys. Rev. E 73, 031108 (2006)

[37] F. Bartumeus and S. A. Levin, Proc. Natl. Acad. Sci. USA 105, 19072 (2008).

[38] O. Bénichou, C. Loverdo, M. Moreau, and R. Voituriez, Rev. Mod. Phys. 83, 81 (2011).

[39] M. A. Lomholt, T. Koren, R. Metzler, and J. Klafter, Proc. Natl. Acad. Sci. USA 105, 11055 (2008).

[40] O. Bénichou, C. Loverdo, M. Moreau, and R. Voituriez, Phys. Rev. E 74, 020102 (2006). 